\title{
Thioridazine elicits potent antitumor effects in colorectal cancer stem cells
}

\author{
CHEN ZHANG, PING GONG, PENGFEI LIU, NING ZHOU, YULAI ZHOU and YI WANG
}

Department of Regenerative Medicine, School of Pharmaceutical Science, Jilin University, Changchun, Jilin 130021, P.R. China

Received July 14, 2016; Accepted December 5, 2016

DOI: $10.3892 /$ or.2016.5313

\begin{abstract}
As a member of the phenothiazine family, thioridazine (THIO) is a potent anti-anxiety and anti-psychotic drug. Recent studies have reported that THIO could suppress the growth of several types of cancer cells. However, the effect of THIO on colorectal cancer stem cells (CSCs) has not been investigated. In the present study, we examined the effect of THIO on viability of CSCs isolated from the human colon cancer cell line HCT116 and its colony-formation ability, along with its stem cell-specific gene expression. The CSCs, $\mathrm{EpCAM}^{+}$and $\mathrm{CD}_{4} 4^{+}$subpopulations from HCT116 cells were isolated using immunomagnetic beads. After incubation with several concentrations of THIO, we evaluated the proliferation and invasion ability of colon CSCs, as well as cell apoptosis. We found that THIO significantly suppressed the proliferation and invasion of colon CSCs and induced cell apoptosis in a concentration-dependent manner. The expression of some apoptosis genes (Bax and caspase-3) was upregulated after treatment with THIO, while that of the anti-apoptosis gene Bcl-2 was downregulated. Moreover, the CSC mitochondrial membrane potential was downregulated. Overall, this study showed that THIO inhibits the proliferation of CSCs derived from the HCT116 cell line through induction of apoptosis, and thus, could be a promising agent for the treatment of colon cancer and worthy of exploring in prospective clinical studies.
\end{abstract}

\section{Introduction}

Thioridazine (10-[2-(1-methyl-2-piperidyl)ethyl]-2-methylthiophenothiazine, THIO), a member of the phenothiazine family, was originally used to treat psychotic disorders such as psychosis and schizophrenia (1-3). In addition, in advanced cancer patients, this drug has been used to treat cancer-related sweating (4,5), as well as depression (6). In recent years, however, some studies have demonstrated that THIO could

Correspondence to: Professor Yi Wang, Department of Regenerative Medicine, School of Pharmaceutical Science, Jilin University, No. 1266 Fujin Road, Changchun, Jilin 130021, P.R. China

E-mail:wangyi@jlu.edu.cn

Key words: thioridazine, colon cancer, cancer stem cells, apoptosis inhibit the growth of some cancer cell lines (7-14). It has also shown selectivity for cancer stem cells (CSCs), such as leukemic cancer stem cells (15), glioblastoma stem cells (3) and breast cancer stem cells (16). Thus, THIO is currently regarded as a drug with potential usefulness in anticancer chemotherapy $(9,17)$.

Recent evidence suggests that cancer development is mainly driven by a rare population of cells, the CSCs $(16,17)$. Moreover, some scientists argue that conventional chemotherapeutics are ineffective against human CSCs (18). Therefore, the development of new drugs targeting CSCs holds special significance in clinical cancer research. Sachlos et al (15) demonstrated that THIO could selectively target neoplastic cells and impair human somatic CSCs capable of in vivo leukemic disease initiation without any effect on normal blood stem cells. Their study identified the potential of THIO to target CSCs directly. However, its exact effect on CSCs of other types of cancers still requires further investigation.

Colon cancer is one of the most prevalent cancers, with incidence rates that have been increasing steadily worldwide $(19,20)$. In recent years, despite a series of remarkable advances in colon cancer chemotherapy, the increased resistance to anticancer drugs has been a serious obstacle to the efficient treatment of the disease. Overcoming drug resistance and targeting CSCs are key for the improvement of chemotherapy response $(15,20,21)$. Therefore, the development of novel effective drugs for colon cancer is urgently required.

In this study, we mainly investigated the anti-proliferation and anti-invasion effects of THIO on CSCs isolated from a human colon cancer cell line (HCT116) and further determined the underlying mechanisms. These findings may contribute to the development of THIO-based chemotherapy for patients with colon cancer resistant to traditional anticancer drugs.

\section{Materials and methods}

Cell lines and culture. The human colon cancer cell line HCT116 and the human lung fibroblast cell line HELF were obtained from the Cell Bank of the Shanghai Institutes for Biological Sciences, Chinese Academy of Sciences (Shanghai, China). HCT116 and HELF cells were maintained in RPMI-1640 medium (Gibco/Thermo Fisher Scientific, Waltham, MA, USA) supplemented with $10 \%$ fetal bovine serum (FBS; Gibco) and penicillin-streptomycin mixed solution. The CSCs isolated from HCT116 cells were cultured in DMEM/F12 medium 
(Gibco) with $10 \mu \mathrm{g} / \mathrm{ml}$ EGF (Sigma-Aldrich, St. Louis, MO, USA), $10 \mu \mathrm{g} / \mathrm{ml}$ bFGF (Sigma-Aldrich) and 1\% B27 supplement (Gibco). The media were changed every other day. The cells were incubated in a humidified incubator with $5 \% \mathrm{CO}_{2}$ at $37^{\circ} \mathrm{C}$, and were passaged by a dilution of $1: 3$ every 4 or 5 days.

EpCAM ${ }^{+}$and $C D 44^{+}$cell selection. The cell selection method followed the protocol published by Zhang et al (21). Briefly, HCT116 cells were rinsed with phosphate-buffered saline (PBS) and detached with trypsin at $37^{\circ} \mathrm{C}$. After centrifugation, cells were first incubated with anti-human CD44 monoclonal antibody conjugated with biotin (eBioscience, Inc., San Diego, CA, USA), and then fractionated using a CELLection Biotin Binder kit (Invitrogen, Carlsbad, CA, USA), according to the manufacturer's recommendations. The isolated cells were further incubated with anti-human EpCAM monoclonal antibody conjugated with biotin (eBioscience) and positive cells were then isolated with the same kit. Cells were cultured in CSC medium, and the purity of such CSCs was evaluated by flow cytometry and immunohistochemistry.

Flow cytometric analysis. The CSCs were dissociated into single cells, further fixed with fixation buffer (eBioscience) and prepared at a concentration of $2.0 \times 10^{6} / \mathrm{ml}$ of PBS. Anti-human CD44 antibody conjugated with FITC (BD Biosciences, San Jose, CA, USA) and anti-human EpCAM antibody conjugated with PE (BD Biosciences) were added and incubated for 30 min at $4^{\circ} \mathrm{C}$. After washing twice with PBS, the cells were acquired and analyzed by FACScalibur (BD Biosciences).

Immunohistochemistry. The single CSCs were seeded into plates covered in Matrigel and cultured for $24 \mathrm{~h}$. They were fixed in $4 \%$ formaldehyde for $20 \mathrm{~min}$ at $4{ }^{\circ} \mathrm{C}$ and permeabilized with $0.1 \%$ Triton X-100 for $10 \mathrm{~min}$ at $25^{\circ} \mathrm{C}$. For immunohistochemistry, the primary antibodies used were anti-human CD44 monoclonal antibodies (1:50; eBioscience) and antihuman EpCAM monoclonal antibodies (1:50; eBioscience). After $12-14 \mathrm{~h}$ of incubation at $4^{\circ} \mathrm{C}$, samples were washed three times with PBS and processed using an ABC kit and DAB solution (both purchased from Vector Laboratories, Inc., Burlingame, CA, USA). Finally, the sections were imaged with an Axio Scope A1 and AxioCAM MRc 5 (Carl-Zeiss, Oberkochen, Germany).

Colony formation assay. The CSCs were dissociated into single cells and seeded into a 96-well plate at a concentration of 0.5 cells/well. Briefly, the cell suspension was diluted at concentration of 50 cells $/ \mathrm{ml}$ and $100 \mu 1$ of cell suspension was added into each well. As a result, there is 1 cell or 0 cell seeded into each well. We observed and imaged cell cloning. The plate was incubated in a humidified incubator with $5 \%$ $\mathrm{CO}_{2}$ at $37^{\circ} \mathrm{C}$ and half of the medium was changed every 3 days. Colonies gradually formed over the next 3 weeks.

Inhibitory effects of THIO on cell proliferation. In order to analyze the anticancer effect of THIO on CSCs derived from HCT116 cells, a CCK-8 assay was performed. In order to explore the effect of THIO on fibroblast cells, representing one of the largest amount of cells in humans, HELF cells were also selected in CCK8-assay. The cells were seeded in 96-well
Table I. Real-time qPCR primer sequences.

\begin{tabular}{ll}
\hline Gene & \multicolumn{1}{c}{ Primer sequence (5'-3') } \\
\hline Nanog & F: ATGCCTGTGATTTGTGGGCC \\
& R: GCCAGTTGTTTTCTGCCAC \\
CLDN-6 & F: GCCAGATGCAGTGCAAGGTGT \\
& R: GATGACAAAGACAATCCCAGAGGTG \\
Bax & F: TAACCAAGGTGCCGGAACTGA \\
& R: GGGAGGAGTCTCACCCAACCA \\
Caspase-3 & F: CATGGAAGCGAATCAATGGACT \\
& R: CTGTACCAGACCGAGATGTCA \\
Bcl-2 & F: GGGGAGGATTGTGGCCTTCTTT \\
& R: TAATGTGCAGGTGCCGGTCAG \\
GAPDH & F: ACCACAGTCCATGCCATCAC \\
& R: TCCACCACCCTGTTGCTGTA
\end{tabular}

F, forward; R, reverse.

plates at a concentration of $1.0 \times 10^{5}$ cells/well, and were treated with THIO (Sigma-Aldrich) at different concentrations $(0,10$, 20 or $50 \mu \mathrm{M}$ ) for $24 \mathrm{~h}$. The medium without any cells was used as the blank group, while $100 \mu \mathrm{M}$ cisplatin (DDP) was used as the positive control group. The proliferation index of each group was determined using the Cell Counting kit-8 (CCK-8; Dojindo Laboratories, Tokyo, Japan) according to the manufacturer's instructions (21). In brief, $10 \mu \mathrm{l}$ of CCK-8 solution was added into each well (containing $100 \mu \mathrm{l}$ of medium) and cultured for $1-2 \mathrm{~h}$ at $37^{\circ} \mathrm{C}$. The absorbance at $450 \mathrm{~nm}$, which was directly proportional to the number of living cells, was observed for each group $(n=4)$. The inhibition ratio was used to measure cell proliferation in the present study and it was described as (absorbance of $0 \mu \mathrm{M}$ group - absorbance of each experimental group)/(absorbance of $0 \mu \mathrm{M}$ group - the absorbance of the blank group).

Cell invasion assay. To assess cell invasion, a Transwell system was used (pore size: $8 \mu \mathrm{m}$; Corning, Inc., Corning, NY, USA) following the manufacturer's protocol. The CSCs were seeded onto the upper insert covered with Matrigel at a concentration of $1 \times 10^{5}$ cells per insert in serum-free medium. Outer wells were filled with RPMI-1640 medium containing $10 \%$ FBS as a chemoattractant. Then, cells were incubated for $48 \mathrm{~h}$ at $37^{\circ} \mathrm{C}$. Non-invading cells were removed by swabbing the top layer, and cells able to migrate through the gel and attach to the lower surface of the membrane were stained with crystal violet. The number of cells in four randomly selected microscopy fields was counted for each filter.

Real-time qPCR. Total RNA was extracted using TRIzol reagent (Invitrogen). For each sample, RNA ( $2 \mathrm{mg}$ ) was reversetranscribed using an RT-PCR kit (Takara, Shiga, Japan), and qPCR was performed with a Thermal Cycler Dice ${ }^{\mathrm{TM}}$ RealTime System and SYBR-Green Premix EX Taq ${ }^{\mathrm{TM}}$ (Takara). In the present study, GAPDH was used for qPCR normalization, and all measurements were performed in triplicate. The primer sequences used $\left(5^{\prime} \rightarrow 3^{\prime}\right)$ are shown in Table I. 


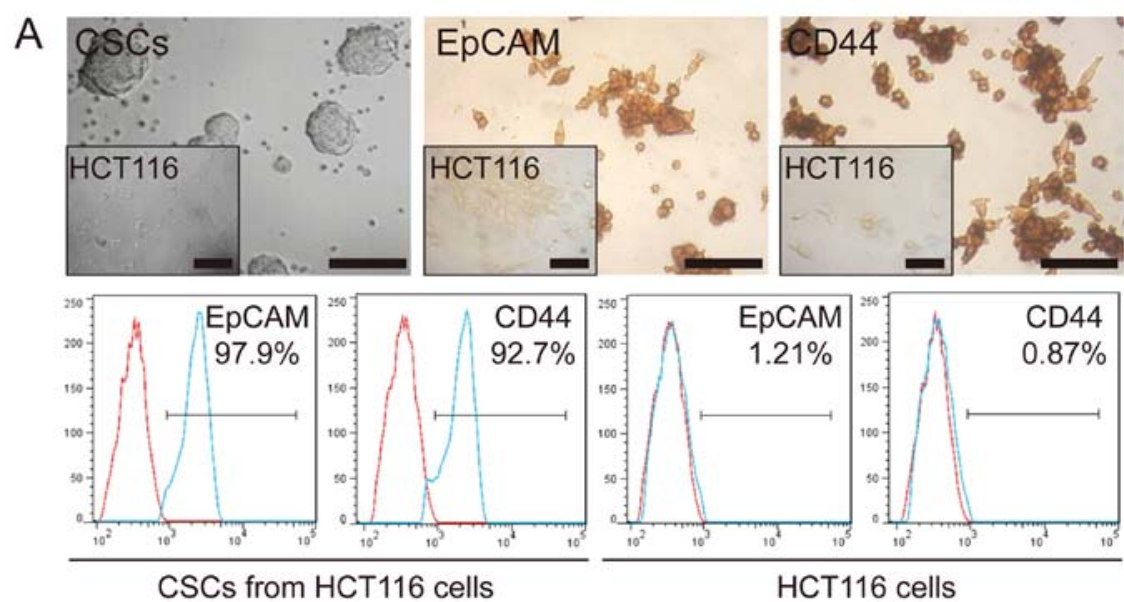

B
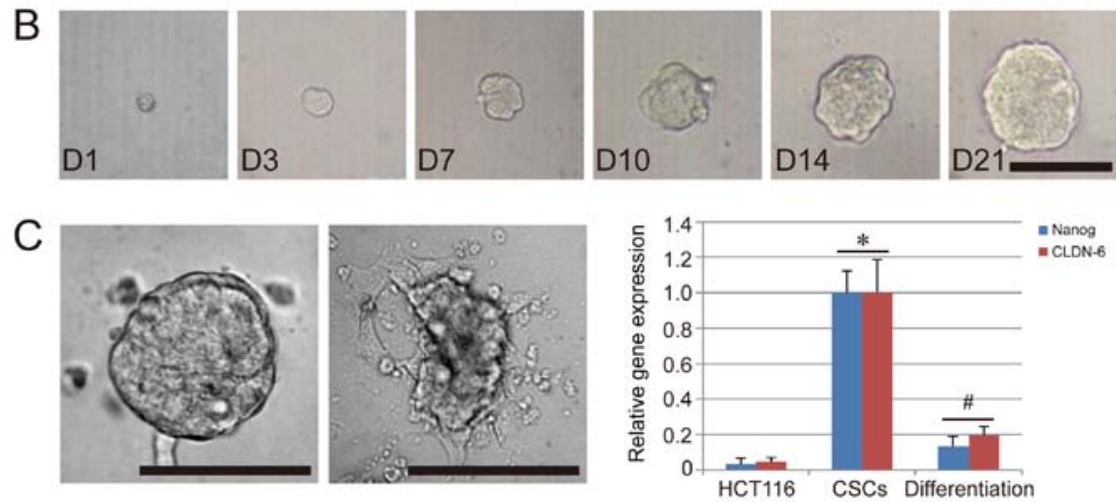

Figure 1. Characterization of CSCs isolated from the HCT116 cell line. (A) Appearance and immune phenotype of isolated CSCs. The CSCs isolated from HCT116 cells formed tumor spheres in suspension when cultured in vitro, and the cells were positive for EpCAM and CD44 under immunohistochemistry and FACS detection. HCT116 cells were used as the negative control group and were nearly negative for EpCAM and CD44. (B) Process of colony formation from one CSC. The CSC proliferated and grew gradually, and formed colonies after 21 days. (C) The necessity of a serum-free culture condition for maintaining stem cell characteristics of CSCs. Cultures under FBS conditions could lead to a layer of adherent confluent cells, as well as the downregulated expression of stem cell-specific genes (NANOG and CLDN-6) in CSCs. Results of qPCR are expressed as mean \pm SEM. A t-test was performed to compare the various groups and $\mathrm{P}<0.05$ was considered statistically significant. ${ }^{~} \mathrm{P}<0.05$ compared with the normal HCT116 group, and ${ }^{\text {"P }}<0.05$ compared with the CSC group without any FBS treatment. Scale bars, $500 \mu \mathrm{m}$.

Western blotting. Western blotting was carried out to test for cleaved caspase- 3 using the same protocol as the one we reported in a previous publication (21). Briefly, cells were lysed with lysis buffer (50 mM Tris pH 7.0, $1 \mathrm{mM}$ EDTA, $150 \mathrm{mM}$ $\mathrm{NaCl}, 1 \% \mathrm{NP} 40,10 \mathrm{mM} \mathrm{NaF}, 1 \mathrm{mM} \mathrm{Na} \mathrm{VO}_{4}$ ) containing protease inhibitor cocktail (Roche, Basel, Switzerland), and protein concentrations were determined using a BCA assay kit (Beyotime Institute of Biotechnology, Nanjing, China). Protein bands were separated by electrophoresis in a $12 \%$ sodium dodecyl sulfate polyacrylamide gel (SDS-PAGE) and electroblotted onto polyvinylidene fluoride (PVDF) membranes (Millipore, Bedford, MA, USA). After blocking them with 4\% non-fat dry milk in Tris-buffered saline (TBS), the membranes were incubated overnight at $4^{\circ} \mathrm{C}$ with primary antibodies (rabbit anti-caspase-3; Santa Cruz Biotechnology, Santa Cruz, CA, USA) diluted 1:1,000 in TBS. They were washed three times with TBS containing $0.5 \%$ Tween-20, and then incubated for $1 \mathrm{~h}$ at $25^{\circ} \mathrm{C}$ with secondary antibodies conjugated with a 1:5,000 dilution of horseradish peroxidase (HRP) in TBS. Membranes were then washed three times in TBS containing $0.5 \%$ Tween- 20 at $25^{\circ} \mathrm{C}$. Finally, protein bands were visualized on X-ray film using enhanced chemiluminescence (ECL; GE Healthcare, Bethesda, MD, USA).
Analysis of cell apoptosis. To analyze cell apoptosis, acridine orange/ethidium bromide (AO/EB) staining and Annexin V-FITC/PI staining were used according to the manufacturer's instructions (BD Biosciences). Moreover, to determine the effect of THIO on mitochondrial membrane potential in CSCs, JC-1 apoptosis detection kit(BDBiosciences) was used according to the manufacturer's instructions and assessed by fluorescence-activated cell sorting (FACS).

Statistical analysis. Statistical analysis was performed with the SPSS 17.0. The results are expressed as mean \pm SEM. The differences between the groups were assessed by one-way ANOVA followed by t-tests. $\mathrm{P}<0.05$ was considered statistically significant.

\section{Results}

Characterization of CSCs from HCT116 cells. The CSCs isolated from HCT116 formed tumor spheres in suspension when cultured in vitro. Their appearance was distinct from the original HCT116 cells, which were spindle-shaped and grew in adherent state (Fig. 1A). After three to four passages, the cells were further characterized by immunohistochemistry 


\section{A}
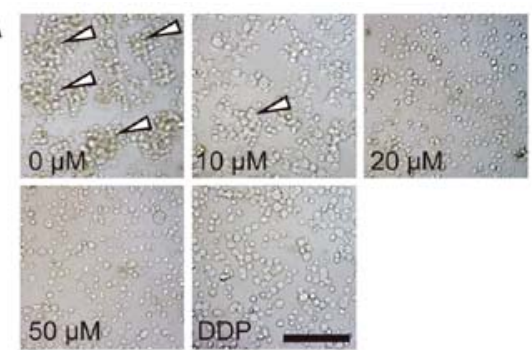

$\mathrm{B}$
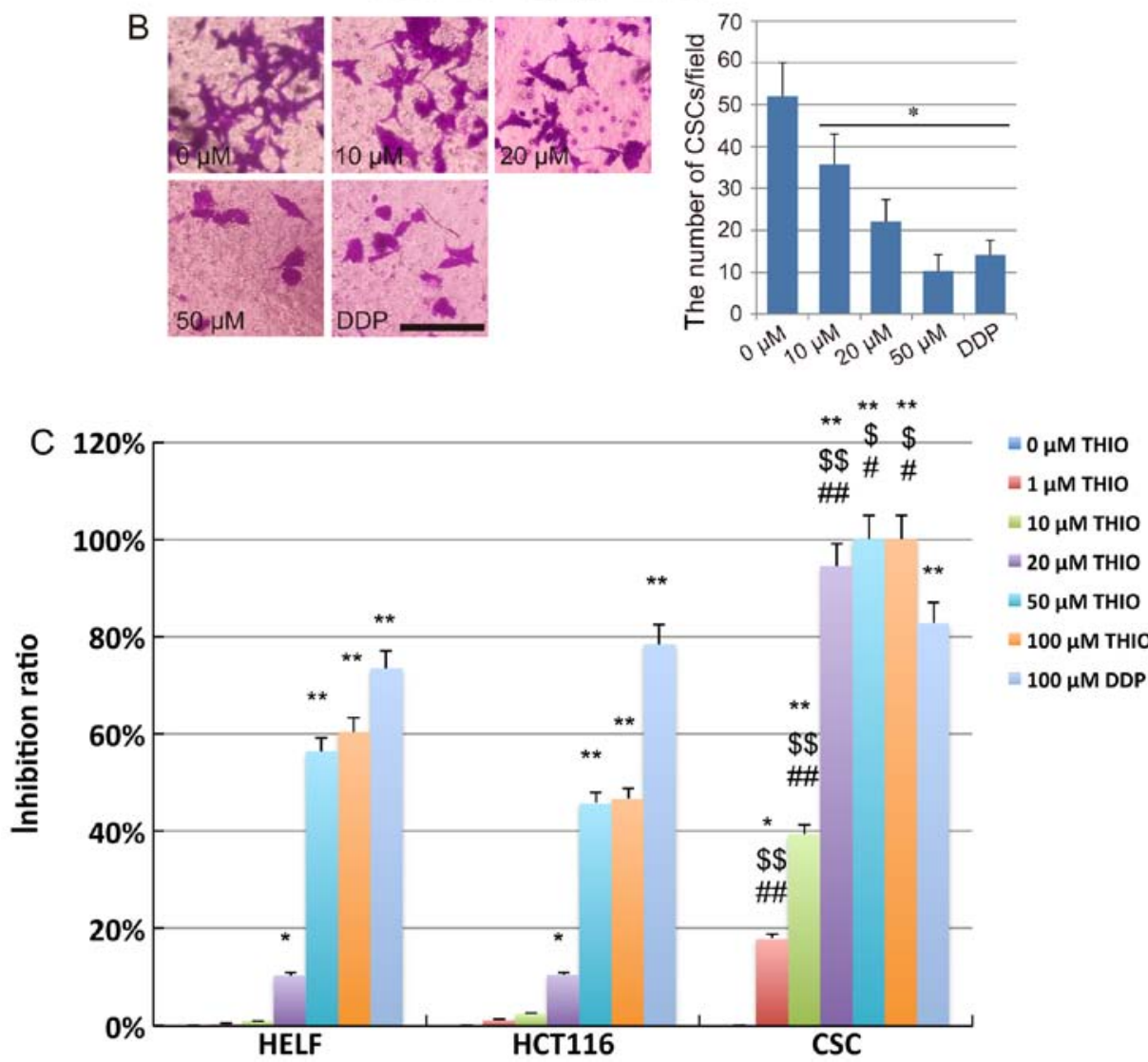

Figure 2. Effect of THIO on the proliferation and invasion of CSCs from HCT116 cells. (A) Effect of THIO on the proliferation ability of CSCs. The morphological alterations of each group are shown, and colony formation is marked by a white arrowhead. (B) Effect of THIO on the invasion ability of CSCs. DDP at a concentration of $100 \mu \mathrm{M}$ was used as the positive drug group. (C) Effect of THIO on the proliferation ability of HELF cells, HCT116 cells and CSCs from HCT116 cells. DDP at a concentration of $100 \mu \mathrm{M}$ was used as the positive drug group. Results are expressed as mean \pm SEM. A t-test was performed to compare the various groups, and $\mathrm{P}<0.05$ was considered statistically significant. ${ }^{*} \mathrm{P}<0.05$ compared with the THIO group at a concentration of $0 \mu \mathrm{M}$, ${ }^{\$} \mathrm{P}<0.05$, and ${ }^{\$ S} \mathrm{P}<0.01$ compared with the THIO group at the same concentration in HELF cells. ${ }^{\#} \mathrm{P}<0.05$ and ${ }^{\# \#} \mathrm{P}<0.01$ compared with the group of THIO at the same concentration in HCT116 cells. Scale bars, $500 \mu \mathrm{m}$.

and flow cytometry. The results indicated that the CSCs used in the present study were positive for EpCAM (97.9\%) and CD44 (92.7\%), while HCT116 cells used as the negative control group displayed a low expression of EpCAM (1.21\%) and CD44 (0.87\%) (Fig. 1A). To further confirm the colonyforming ability of the CSCs, we separated the CSC spheres into single cells and analyzed their colony-formation ability in 96-well plates. As expected, a single CSC could proliferate and grow; in fact, over $40 \%$ of the CSCs formed a tumor sphere after 21 days of single-cell culture (Fig. 1B). In addition, we confirmed that the serum-free condition was necessary for the colony-formation ability and the stem cell-specific gene expression of CSCs. We compared the in vitro cell culture in basal conditions plus 10\% FBS and in serum-free conditions. Cultures under FBS conditions could lead to a layer of adherent confluent cells. Compared with HCT116 cells, the
CSCs showed a high expression of stem cell-specific genes, NANOG, and CLDN-6 $(\mathrm{P}<0.05)$, while downregulation of these genes occurred in the CSCs cultured under FBS conditions $(\mathrm{P}<0.05)($ Fig. 1C). These results suggest that the tumor sphere-like colonies could be obtained from the HCT116 cell line, and that these cells had some stem cell characteristics. In serum-free medium supplemented with EGF and bFGF, the CSCs differentiated even under conditions of an extra-low cell concentration, such as single cell conditions, ruling out the possibility that CSCs may aggregate owing to the high concentration of cells in cultures.

Effect of THIO on the proliferation and invasion of CSCs from HCT116 cells. After treatment with THIO for $24 \mathrm{~h}$ at different concentrations, severe morphological alterations were observed in the majority of CSCs cells. We found that the 
A
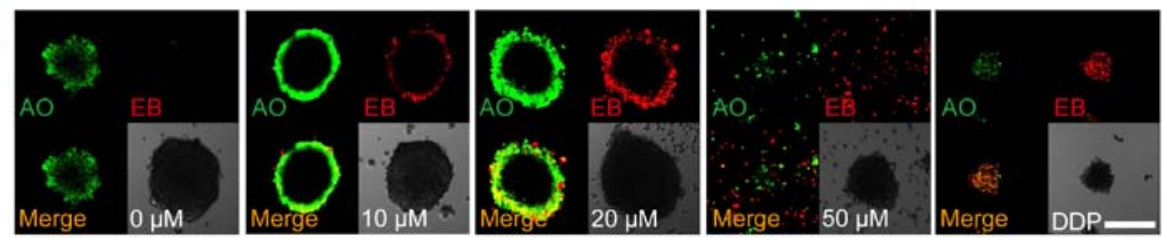

B

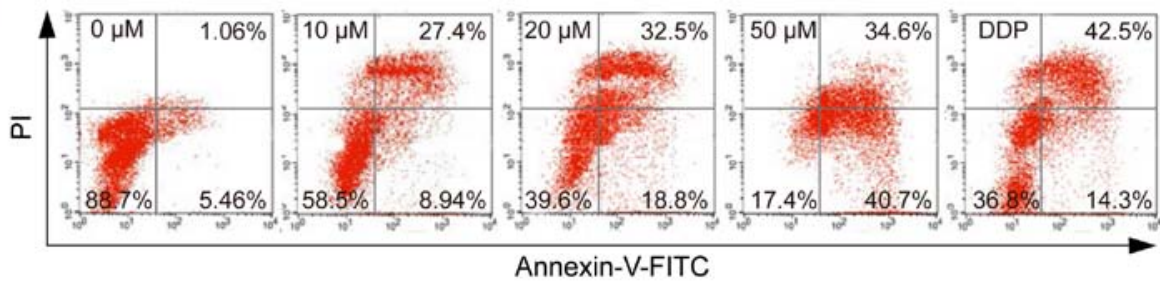

$\mathrm{C}$
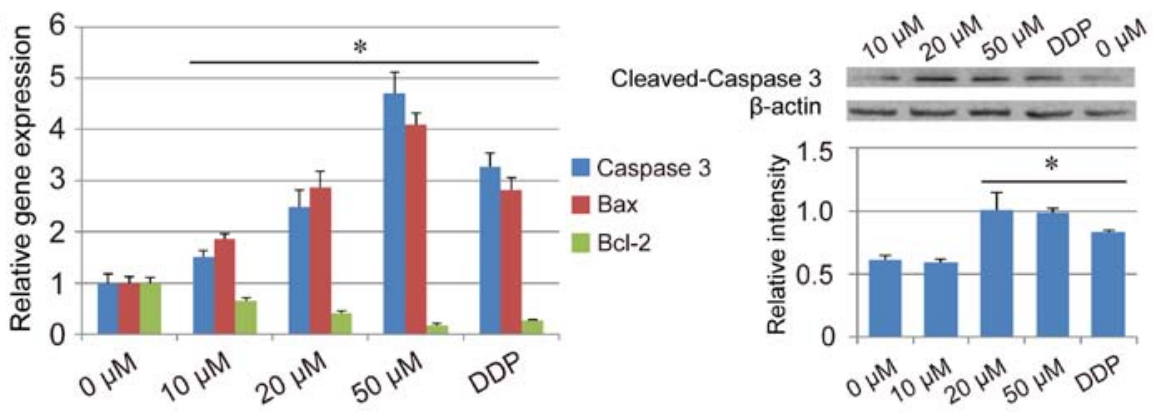

$\mathrm{D}$

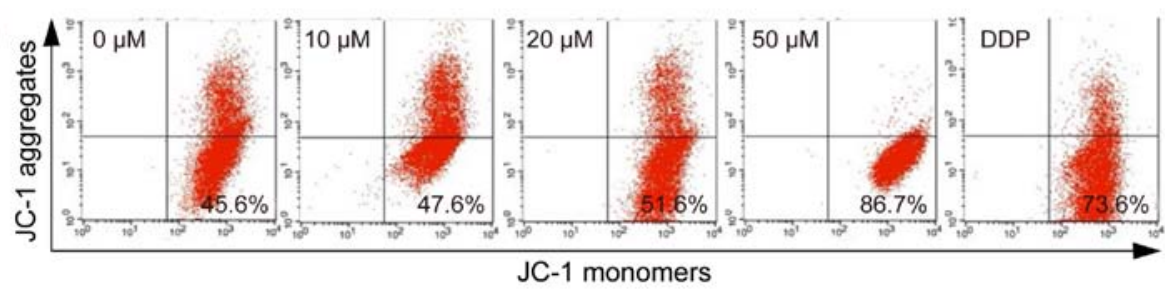

Figure 3. The effect of THIO on apoptosis in CSCs. (A) Results of AO/EB double-staining. After treatment with THIO at 0 , 10,20 and $50 \mu \mathrm{M}$, the tumor spheres were stained with AO (red)/EB (green) and observed under confocal microscopy. Scale bar, $200 \mu \mathrm{m}$. (B) Annexin V/PI double staining analysis of THIO-treated CSCs. Annexin V was labeled with FITC. The early-stage apoptotic cells (Annexin V-positive and PI-negative cells), as well as late-stage apoptotic cells (Annexin V-positive and PI-positive cells), in CSCs were analyzed with FACS. (C) Expression of apoptosis and anti-apoptosis genes and protein in CSCs treated with THIO. Relative gene expression results are shown on the left, while western blot results are shown on the right. Results are expressed as mean \pm SEM. A t-test was performed to compare the various groups and $\mathrm{P}<0.05$ was considered statistically significant. * $\mathrm{P}<0.05$ compared with the group of THIO at a concentration of $0 \mu \mathrm{M}$. (D) THIO induces cell apoptosis in CSCs via the mitochondrial pathway. JC-1 staining was used to determine the loss of mitochondrial membrane potential in CSCs. Values in the right lower quadrant indicate the proportion of cells with loss of mitochondrial membrane potential.

CSCs could still form tumor sphere-like colonies when treated with THIO at $10 \mu \mathrm{M}$. However, the number of colonies formed at $10 \mu \mathrm{M}$ was considerably lower than at $0 \mu \mathrm{M}$. No colony formation could be observed at THIO concentrations of $20 \mu \mathrm{M}$ and above and after treatment with DDP (Fig. 2A).

In vitro invasion assays were performed using CSCs from HCT116 cells treated with THIO in a Transwell system. After crystal violet staining, we observed that the CSC invasion across the membrane from the upper chamber to the lower surface of the membrane was suppressed by THIO. The number of cells on the lower surface of the membrane clearly decreased in the THIO-treated groups at concentrations of 20 and $50 \mu \mathrm{M}$, while a slight inhibition of the cell invasion ability was observed in the $10 \mu \mathrm{M}$ concentration group (Fig. 2B). After counting the number of cells on the lower surface of the membrane, we concluded that THIO could significantly suppress the invasion of CSCs $(\mathrm{P}<0.05)$. This effect occurred in a concentration-dependent manner $(52.0 \pm 8.0$ cells for the $0 \mu \mathrm{M}$ group, $35.6 \pm 7.3$ cells for the $10 \mu \mathrm{M}$ group, $22.0 \pm 5.4$ cells for the $20 \mu \mathrm{M}$ group, $10.3 \pm 4.0$ cells for the $50 \mu \mathrm{M}$ group, and 14.3 \pm 3.3 cells for the DDP group) (Fig. 2B).

To further analyze the anticancer effect of THIO on CSCs derived from HCT116 cells, a CCK-8 assay was performed using treated cells (normal human lung fibroblast cell line, HELF cells, HCT116 cells and CSCs). The results indicated that the proliferation of HELF and HCT116 cells was significantly inhibited by THIO at 50 and $100 \mu \mathrm{M}$, while that of CSCs was significantly inhibited by THIO at a concentration as low as $1 \mu \mathrm{M}$. The inhibition ratio of THIO at 1 and $10 \mu \mathrm{M}$ was 19.2 and $39.5 \%$, respectively. However, THIO concentrations of 20,50 and $100 \mu \mathrm{M}$ exhibited inhibition at very high rates (94.5, 100 and $100 \%$, respectively). The positive control group (10 $\mu \mathrm{M}$ DDP) showed an inhibition rate of $82.7 \%$. Interestingly, THIO at $20 \mu \mathrm{M}$ did affect the vitality of HELF cells, but the inhibition rate was only $10.6 \%$, while that of CSCs was $94.5 \%$ (Fig. 2C). These results indicate that, at a suitable concentration, THIO may be an optimal novel agent for colon cancer treatment. However, further studies are necessary to elucidate the mechanism of action driving this anticancer activity. 
Analysis of THIO-induced apoptosis in CSCs. THIO has been reported to induce cervical and endometrial cancer cell apoptosis (7). To detect whether THIO could induce apoptosis in CSCs derived from HCT116 cells, we carried out AO/EB staining. The CSC spheres were labeled by $\mathrm{AO} / \mathrm{EB}$ after treatment with THIO at different concentrations, and dual staining was examined using confocal microscopy. No significant apoptosis was detected in the negative control group. However, in the group treated with THIO at a concentration of $10 \mu \mathrm{M}$, many early-stage apoptotic cells (marked by yellow-green AO nuclear staining) and some late-stage apoptotic cells (marked by orange-red nuclear EB staining) were detected. At higher concentrations (20 and $50 \mu \mathrm{M}$ ), the number of late-stage apoptotic cells was even higher (Fig. 3A). At $50 \mu \mathrm{M}$, CSC spheres were much smaller than at lower concentrations, and many single cells could be observed in late-stage apoptosis (Fig. 3A). Further detection by the Annexin V/PI double staining assay revealed that THIO treatment could lead to an increase in the proportion of early-stage apoptotic cells (Annexin V-positive and PI-negative cells), as well as late-stage apoptotic cells (Annexin V-positive and PI-positive cells) in CSCs (Fig. 3B). Further analysis indicated that this induction of apoptosis by THIO occurred in a concentration-dependent manner. The percentage of early-stage apoptotic cells was 5.46, 8.94, 18.8, 40.7 and $14.3 \%$ at THIO concentrations of $0,10,20$ and $50 \mu \mathrm{M}$, and DDP treatment, respectively. The percentage of late-stage apoptotic cells was $1.06,27.4,32.5,34.6$ and $42.5 \%$ at THIO concentrations of $0,10,20$ and $50 \mu \mathrm{M}$, and DDP treatment, respectively (Fig. 3B).

To elucidate the mechanisms of cell apoptosis induced by THIO, cell apoptosis genes and mitochondrial membrane potential were assayed as part of this study. qPCR results indicated that apoptosis genes such as caspase- 3 and Bax were significantly upregulated in THIO-treated CSCs $(\mathrm{P}<0.05)$, while the anti-apoptosis gene $\mathrm{Bcl}-2$ was significantly downregulated under the same conditions $(\mathrm{P}<0.05)$ (Fig. 3C). Moreover, the expression of the caspase-3 gene was confirmed by the western blot analysis. To evaluate the THIO-induced cell apoptosis at the subcellular organelle level, JC-1 staining was carried out to determine the alterations in mitochondria. The percentage of cells with loss of mitochondrial membrane potential increased in a concentration-dependent manner (45.6\% for the $0 \mu \mathrm{M}$ group, $47.6 \%$ for the $10 \mu \mathrm{M}$ group, $51.6 \%$ for the $20 \mu \mathrm{M}$ group, $86.7 \%$ for the $50 \mu \mathrm{M}$ group, and $73.6 \%$ for the DDP group) (Fig. 3D). The results suggest that THIO may induce apoptosis in CSCs from HCT116 cells via the loss of mitochondrial membrane potential.

\section{Discussion}

THIO has been used in human clinical studies for over 50 years, especially for tuberculosis (TB) therapy $(22,23)$, alleviation of schizophrenia (24) and reduction of pain in cancer (25). In recent years, THIO has been reported to suppress cell proliferation and induce cell apoptosis in several types of cancers $(2,7,9,26,27)$. However, the cytotoxic effect of THIO on colon cancer has not been examined, and the effects of THIO on the viability of CSCs, which are responsible for apoptosis resistance, self-renewal and differentiation, should be investigated thoroughly. The present study revealed that THIO could reduce the viability of CSCs from colon cancer cells (HCT116) and induce apoptosis of CSCs via the mitochondrial pathway. Previous studies in our group revealed that THIO also induces apoptosis in CSCs derived from other cancer cell lines, such as DU145 (human prostate carcinoma cell line) (data not shown). Thus, THIO-based chemotherapy may prove useful in the treatment of various types of cancers. This must be evaluated in future clinical studies.

Although the anticancer effect of THIO has been demonstrated in vivo using mouse models $(26,28,29)$, few clinical trials have been carried out in cancer patients (30) for the complex mechanisms of the THIO anticancer effect to be clearly understood. In the present study, JC-1 results showed that the mitochondrial membrane potential in CSCs was downregulated during apoptosis, which indicates that THIOinduced apoptosis in CSCs may be related to the mitochondrial pathway. In addition to the direct cytotoxic effect on cancer cells, THIO can potentially induce CSC differentiation to overcome neoplastic self-renewal, and compel CSCs to enter the normal cellular lifecycle, via antagonism of D2-family DRs differentially expressed in CSCs $(15,31)$. However, this theory may only apply to a therapy targeting CSCs, and the effect of THIO on CSCs has so far been demonstrated only in human leukemia and breast cancer studies $(15,16)$. Therefore, the specific effects of THIO on CSCs from other cancers, as well as the associated mechanisms, still require exploration.

THIO can prevent the exclusion of some small molecules from cancer cells $(26,32,33)$. This may explain the anti-CSC ability of THIO that was discovered through small-molecule library screening $(26,34)$. A previous study has indicated that THIO could reverse chemoresistance of cancer cells and achieve a significant therapeutic outcome in combination therapy with verapamil (35). A recent study also demonstrated that the combination of THIO and doxorubicin using polymeric micelles might provide a promising strategy for breast cancer treatment by targeting both cancer cells and cancer stem cells (16). Thus, THIO may be useful as a type of adjuvant in combination with other chemotherapeutic drugs.

In clinical studies of cancer, THIO is mainly used for managing depression and psychosis (30). Apart from understanding the therapeutic mechanisms, it is important to consider the optimal dose in order to avoid serious sideeffects, which can include movement disorders and cardiac and central nervous system toxicity. Thus, the potential toxic effects of high-dose THIO treatments should be evaluated carefully.

Conclusion, this research showed that THIO could suppress proliferation, reduce invasion, and induce apoptosis in CSCs via the mitochondrial pathway in a concentration-dependent manner. Even though the anticolon cancer effect of THIO still needs to be evaluated in vivo, and in well-designed human clinical trials, there is enough evidence to suggest that THIO may be a promising novel agent as an adjuvant for the treatment of colon cancer, and possibly other cancers.

\section{Acknowledgements}

The present study was supported by the Jilin Province Science Foundation (20120960 and 20160204036YY). 


\section{References}

1. Zhelev Z, Ohba H, Bakalova R, Hadjimitova V, Ishikawa M, Shinohara $Y$ and Baba Y: Phenothiazines suppress proliferation and induce apoptosis in cultured leukemic cells without any influence on the viability of normallymphocytes. Phenothiazines and leukemia. Cancer Chemother Pharmacol 53: 267-275, 2004

2. Min KJ, Seo BR, Bae YC, Yoo YH and Kwon TK: Antipsychotic agent thioridazine sensitizes renal carcinoma Caki cells to TRAIL-induced apoptosis through reactive oxygen speciesmediated inhibition of Akt signaling and downregulation of Mcl-1 and c-FLIP(L). Cell Death Dis 5: e1063, 2014.

3. Cheng HW, Liang YH, Kuo YL, Chuu CP, Lin CY, Lee MH, Wu AT, Yeh CT, Chen EI, Whang-Peng J, et al: Identification of thioridazine, an antipsychotic drug, as an antiglioblastoma and anticancer stem cell agent using public gene expression data. Cell Death Dis 6: e1753, 2015.

4. Cowap J and Hardy J: Thioridazine in the management of cancerrelated sweating. J Pain Symptom Manage 15: 266, 1998.

5. Zhukovsky DS: Fever and sweats in the patient with advanced cancer. Hematol Oncol Clin North Am 16: 579-588, viii, 2002. viii.

6. Ly KL, Chidgey J, Addington-Hall J and Hotopf M: Depression in palliative care: A systematic review. Part 2. Treatment. Palliat Med 16: 279-284, 2002.

7. Kang S, Dong SM, Kim BR, Park MS, Trink B, Byun HJ and Rho SB: Thioridazine induces apoptosis by targeting the PI3K/ $\mathrm{Akt} / \mathrm{mTOR}$ pathway in cervical and endometrial cancer cells. Apoptosis 17: 989-997, 2012.

8. Rho SB, Kim BR and Kang S: A gene signature-based approach identifies thioridazine as an inhibitor of phosphatidylinositol3'-kinase (PI3K)/AKT pathway in ovarian cancer cells. Gynecol Oncol 120: 121-127, 2011.

9. Choi AR, Kim JH and Yoon S: Thioridazine specifically sensitizes drug-resistant cancer cells through highly increase in apoptosis and P-gp inhibition. Tumour Biol 35: 9831-9838, 2014.

10. Lu M, Li J, Luo Z, Zhang S, Xue S, Wang K, Shi Y, Zhang C, Chen $\mathrm{H}$ and $\mathrm{Li} \mathrm{Z}$ : Roles of dopamine receptors and their antagonist thioridazine in hepatoma metastasis. Onco Targets Ther 8: $1543-1552,2015$

11. Gong L, Wang Y, Tong S, Liu L, Niu L, Yuan Y and Bao Y: Mechanism of killing effect of thioridazine on human lung cancer PC9 cells. Zhongguo Fei Ai Za Zhi 18: 727-733, 2015 (In Chinese).

12. Yin T, He S, Shen G, Ye T, Guo F and Wang Y: Dopamine receptor antagonist thioridazine inhibits tumor growth in a murine breast cancer model. Mol Med Rep 12: 4103-4108, 2015.

13. Meng Q, Sun X, Wang J and Wang Y: Mechanism of thioridazine plus medroxyprogesterone in the treatment of endometrial cancer Zhonghua Yi Xue Za Zhi 95: 1540-1543, 2015 (In Chinese).

14. Liu JK, Hao YJ, Huang JW, Li X, Cai HB and Peng K: Mechanism of thioridazine-induced apoptosis of human colorectal cancer SW480 cells. Nan Fang Yi Ke Da Xue Xue Bao 35: 511-515, 2015 (In Chinese).

15. Sachlos E, Risueño RM, Laronde S, Shapovalova Z, Lee JH, Russell J, Malig M, McNicol JD, Fiebig-Comyn A, Graham M, et al: Identification of drugs including a dopamine receptor antagonist that selectively target cancer stem cells. Cell 149: 1284-1297, 2012.

16. Ke XY, Lin Ng VW, Gao SJ, Tong YW, Hedrick JL and Yang YY: Co-delivery of thioridazine and doxorubicin using polymeric micelles for targeting both cancer cells and cancer stem cells. Biomaterials 35: 1096-1108, 2014

17. Dick JE: Looking ahead in cancer stem cell research. Nat Biotechnol 27: 44-46, 2009.

18. Guan Y, Gerhard B and Hogge DE: Detection, isolation, and stimulation of quiescent primitive leukemic progenitor cells from patients with acute myeloid leukemia (AML). Blood 101: 3142-3149, 2003.
19. Jemal A, Siegel R, Ward E, Hao Y, Xu J, Murray T and Thun MJ: Cancer statistics, 2008. CA Cancer J Clin 58: 71-96, 2008.

20. Kim EJ, Kang JI, Kwak JW, Jeon CH, Tung NH, Kim YH, Choi CH, Hyun JW, Koh YS, Yoo ES, et al: The anticancer effect of (1S,2S,3E,7E,11E)-3,7,11, 15-cembratetraen-17,2-olide(LS-1) through the activation of TGF- $\beta$ signaling in SNU-C5/5-FU, fluorouracil-resistant human colon cancer cells. Mar Drugs 13: 1340-1359, 2015

21. Zhang C, Tian Y, Song F, Fu C, Han B and Wang Y: Salinomycin inhibits the growth of colorectal carcinoma by targeting tumor stem cells. Oncol Rep 34: 2469-2476, 2015.

22. Amaral L, Kristiansen JE, Abebe LS and Millett W: Inhibition of the respiration of multi-drug resistant clinical isolates of Mycobacterium tuberculosis by thioridazine: Potential use for initial therapy of freshly diagnosed tuberculosis. J Antimicrob Chemother 38: 1049-1053, 1996.

23. Boeree MJ: Global clinical trials for the treatment of TB with thioridazine. Recent Pat Antiinfect Drug Discov 6: 99-103, 2011.

24. Kleibel F: A method of alleviation of pain in cancer patients. Clinical trial of thioridazine (Melleril and Mellerettes) in 251 patients. Munch Med Wochenschr 103: 2341-2343, 1961 (In German)

25. Smith RC, Baumgartner R, Burd A, Ravichandran GK and Mauldin M: Haloperidol and thioridazine drug levels and clinical response in schizophrenia: Comparison of gas-liquid chromatography and radioreceptor drug level assays. Psychopharmacol Bull 21: 52-58, 1985.

26. Mu J, Xu H, Yang Y, Huang W, Xiao J, Li M, Tan Z, Ding Q, Zhang L, Lu J, et al: Thioridazine, an antipsychotic drug, elicits potent antitumor effects in gastric cancer. Oncol Rep 31: 2107-2114, 2014

27. Tuynder M, Fiucci G, Prieur S, Lespagnol A, Géant A, Beaucourt S, Duflaut D, Besse S, Susini L, Cavarelli J, et al: Translationally controlled tumor protein is a target of tumor reversion. Proc Natl Acad Sci USA 101: 15364-15369, 2004.

28. Basta-Kaim A, Budziszewska B, Jagła G, Nowak W, Kubera M and Lason W: Inhibitory effect of antipsychotic drugs on the Con A- and LPS-induced proliferative activity of mouse splenocytes: A possible mechanism of action. J Physiol Pharmacol 57: 247-264, 2006

29. Park MS, Dong SM, Kim BR, Seo SH, Kang S, Lee EJ, Lee SH and Rho SB: Thioridazine inhibits angiogenesis and tumor growth by targeting the VEGFR-2/PI3K/mTOR pathway in ovarian cancer xenografts. Oncotarget 5: 4929-4934, 2014.

30. Hercbergs A: Thioridazine: A radiation enhancer in advanced cervical cancer? Lancet 2: 737, 1988.

31. Gatto F and Hofland LJ: The role of somatostatin and dopamine D2 receptors in endocrine tumors. Endocr Relat Cancer 18: R233-R251, 2011.

32. Akiyama S, Shiraishi N, Kuratomi Y, Nakagawa M and Kuwano M: Circumvention of multiple-drug resistance in human cancer cells by thioridazine, trifluoperazine, and chlorpromazine. J Natl Cancer Inst 76: 839-844, 1986.

33. Efferth T and Volm M: Reversal of doxorubicin-resistance in sarcoma 180 tumor cells by inhibition of different resistance mechanisms. Cancer Lett 70: 197-202, 1993.

34. Sutton LP and Rushlow WJ: The dopamine D2 receptor regulates Akt and GSK-3 via Dvl-3. Int J Neuropsychopharmacol 15: 965-979, 2012

35. Castaing M, Loiseau A and Cornish-Bowden A: Synergy between verapamil and other multidrug -resistance modulators in model membranes. J Biosci 32: 737-746, 2007. 